\title{
Progressive multifocal leukoencephalitis in a patient with sarcoidosis on hydroxychloroquine with negative cerebrospinal fluid testing for the John Cunningham virus
}

\author{
Ovais Inamullah', Casey Farin ${ }^{1}$, Linh Tran², Roger McLendon ${ }^{3}$, Mark Skeen ${ }^{1}$, Joel Morgenlander ${ }^{1}$ \\ ${ }^{1}$ Neurology Department, Duke University Hospital, Durham, NC 27705, USA. \\ ${ }^{2}$ Child Neurology Department, Duke University Hospital, Durham, NC 27705, USA. \\ ${ }^{3}$ Pathology Department, Duke University Hospital, Durham, NC 27705, USA.
}

Correspondence to: Dr. Ovais Inamullah, Neurology Department, Duke University Hospital, 2301 Erwin Rd, Durham, NC 27705, USA. E-mail: ovi@duke.edu

\begin{abstract}
How to cite this article: Inamullah O, Farin C, Tran L, McLendon R, Skeen M, Morgenlander J. Progressive multifocal leukoencephalitis in a patient with sarcoidosis on hydroxychloroquine with negative cerebrospinal fluid testing for the John Cunningham virus. Neuroimmunol Neuroinflammation 2018;5:3. http://dx.doi.org/10.20517/2347-8659.2017.61
\end{abstract}

Received: 29 Nov 2017 First Decision: 22 Jan 2018 Revised: 24 Jan 2018 Accepted: 29 Jan 2018 Published: 31 Jan 2018

Science Editor: Athanassios P. Kyritsis Copy Editor: Jun-Yao Li Production Editor: Huan-Liang Wu

\begin{abstract}
Progressive multifocal leukoencephalopathy $(\mathrm{PML})$ is a severe demyelinating disease of the central nervous system caused by the John Cunningham (JC) virus typically seen in immuno-compromised patients. Several drugs that suppress that immune system have already been known to cause PML such as natalizumab and rituximab. We present a patient with sarcoidosis who develops PML in the rare setting of minimal immunosuppression with only hydroxychloroquine. There was significant delay in the diagnosis due to negative cerebrospinal fluid testing for JC virus and concern for neuro-sarcoidosis, but eventually a diagnosis of PML was made via brain biopsy.
\end{abstract}

Keywords: Progressive multifocal leukoencephalopathy, sarcoidosis, hydroxychloroquine, neuroimmunology

\section{INTRODUCTION}

Progressive multifocal leukoencephalopathy (PML) is a severe demyelinating disease of the central nervous system caused by the John Cunningham (JC) virus. Approximately $86 \%$ of adults have antibodies to the JC virus, believed to be secondary to an asymptomatic childhood infection ${ }^{[1]}$. When a patient becomes

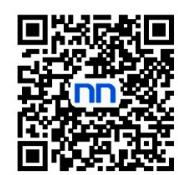


immunosuppressed, the JC virus can spread to the central nervous system, and cause a life-threatening severe neurological illness.

Patients with PML usually have gradually worsening neurological deficits including encephalopathy, motor deficits, and visual symptoms. PML lesions are generally in the white matter, and magnetic resonance imaging (MRI) typically shows that these lesions have no mass effect and no contrast enhancement. Diagnosis can be established by confirming JC virus DNA in the cerebrospinal fluid (CSF) along with typical imaging, but definitive diagnosis requires brain biopsy with the histopathologic triad of demyelination, bizarre astrocytes, and enlarged oligodendroglial nuclei coupled with techniques to show the presence of JC virus $^{[2]}$. PML is an incredibly rare condition. It occurs almost always in patients with a suppressed immune system, either from medications or a preexisting illness such as human immunodeficiency virus (HIV), chronic lymphocytic leukemia, or lymphoma. Several immune suppressing medications have been known to cause PML, most notably natalizumab and rituximab. Hydroxychloroquine (also known as plaquenil) is a medication frequently used to suppress the immune system that has not been previously associated with PML.

\section{CASE REPORT}

Patient D is a 65-year-old man with a history of asthma, benign prostatic hyperplasia, pre-diabetes, and dermatologic sarcoidosis who presented to the Duke General Neurology service in July of 2017 with about 6 months of rapidly worsening right-sided weakness, facial weakness, and cognitive decline. He had been taking hydroxychloroquine $200 \mathrm{mg}$ daily to treat his dermatologic sarcoidosis since September of 2014 (about 3 years prior to presentation). No other immunosuppressive drugs have been shown in medical records. His other home medications were paxil, aspirin, vitamin D, montelukast, and flomax. His rheumatologist also gave him a prolonged steroid taper after his symptoms first began.

In mid-March, he initially noticed right foot numbness and weakness and went to his podiatrist and primary care doctor, who both documented normal neurologic examinations. In early April, the patient was seen by his rheumatologist, who reported right foot drop, prescribed an oral steroid course, and referred him to a neurologist, who documented weakness and decreased sensation to pinprick in the distal right lower extremity. In early May, he was noted to have involvement of his proximal right lower extremity, with mild weakness of hip flexion and extension, moderate weakness of knee flexion and extension, and almost complete inability to dorsiflex or plantarflex his right foot. In early July, he followed up with his neurologist and reported continued progression of weakness, including his right arm. On examination, he had a right pronator drift with mild weakness of his right hand intrinsic muscles and difficulty with fine motor skills, as well as mild weakness of hip flexion, moderate weakness of knee flexion and extension, and severe weakness of dorsiflexion and plantarflexion on his right side. He was walking with a walker and dragging his right foot, and he had fallen several times. He was also noted to have flattening of his right nasolabial fold.

Due to his multifocal and rapidly progressive motor symptoms, his preliminary diagnosis was a variant of motor neuron disease. He initially received a broad laboratory work-up, which was unimpressive including negative testing for HIV. Next, due to concern for motor neuron disease, electromyography was performed and showed increased insertional activity in the majority of muscle groups tested with generalized reduction in activation, concerning for a central nervous system etiology. Lumbar puncture was performed and was unremarkable, including negative JC virus polymerase chain reaction (PCR). MRI's of the cervical, thoracic, and lumbar spines were normal. Brain MRI performed revealed enlargement of non-enhancing subcortical and periventricular T2 hyperintensities [Figure 1] with Wallerian degeneration [Figure 2].

There was concern for neuro-sarcoidosis due to his history of dermatologic sarcoidosis despite the lack of contrast enhancement on the MRI lesions. Computed tomography scan of the chest revealed supraclavicular, 


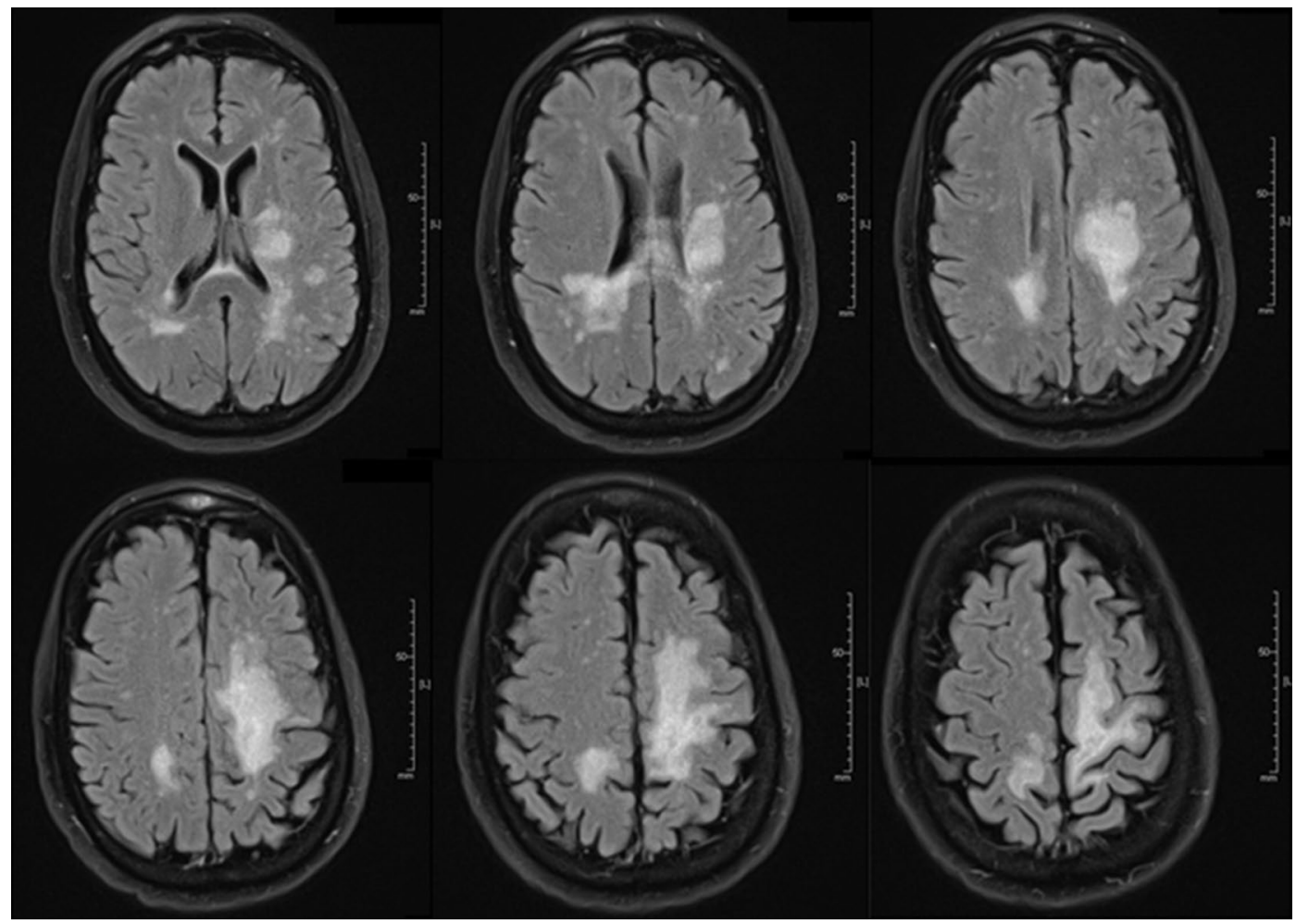

Figure 1. Fluid-attenuated inversion recovery brain magnetic resonance imaging (8 Sep 2017). Confluent periventricular and subcortical white matter lesions seen bilaterally

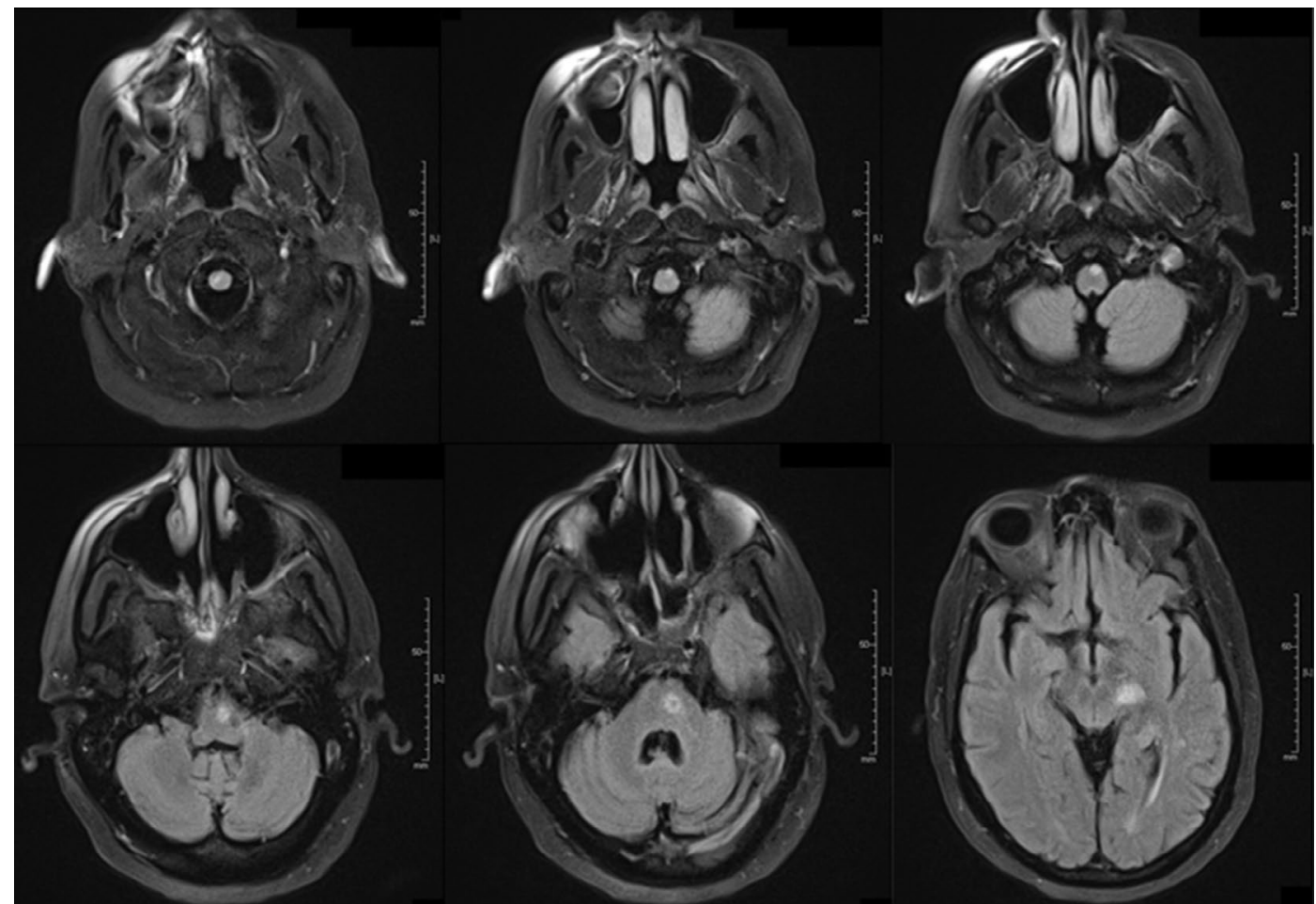

Figure 2. Fluid-attenuated inversion recovery brain magnetic resonance imaging (8 Sep 2017). Prominent Wallerian degeneration noted with lesion located in motor tract starting in the posterior limb of the internal capsule and extending inferiorly, decussating in the inferior medulla

mediastinal, and hilar lymphadenopathy, and lymph node biopsy performed was suggestive of sarcoidosis. He was treated aggressively with intravenous steroids, but unfortunately continued to deteriorate rapidly. He was discharged to a rehab facility but quickly returned with worsening symptoms. 


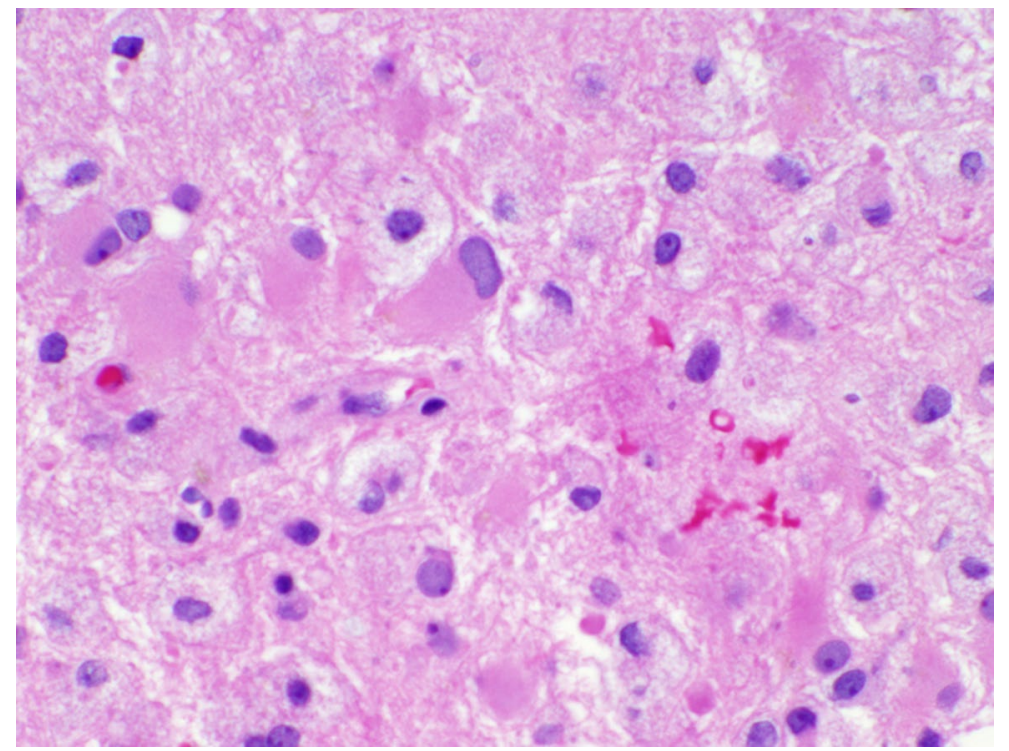

Figure 3. The HE stained section $(\times 40)$ reveals scattered glial cells with enlarged, pleomorphic nuclei with ground glass chromatin, typical of an active progressive multifocal leukoencephalopathy infection

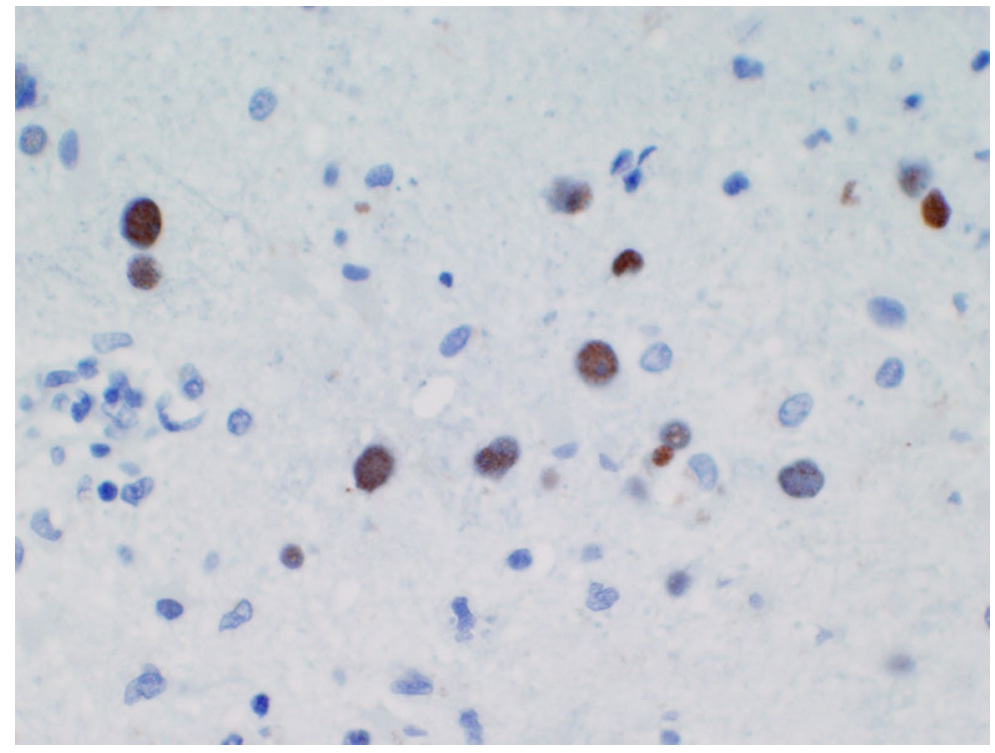

Figure 4. This SV40 immunohistochemical stain $(\times 40)$ reveals the scattered pleomorphic glial cell nuclei to stain strongly due to a crossreactivity among the polyoma viruses

Due to his continued decline, PML was considered as an alternative to neuro-sarcoidosis and stereotactic brain biopsy was performed. Pathology revealed extensive gliosis, abundant lipid-laden macrophages, and large ground glass viral inclusions [Figure 3]. SV40 was immunoreactive in the cells with viral cytopathologic changes confirming a diagnosis of progressive multifocal leukoencephalopathy [Figure 4]. The poor prognosis was discussed with the family and they decided to transition him to comfort care. The patient was discharged home with hospice and died in late September of 2017.

\section{DISCUSSION}

The patient presented is a unique case of PML for three reasons. First, CSF testing was negative for JC Virus DNA. This caused a major delay in the diagnosis. Previous cases have been described where CSF PCR testing 
for JC virus was negative, but brain biopsy supported the diagnosis of $\mathrm{PML}^{[3]}$. Typically this happens if the CSF testing is done very early, but this case was different in that patient D had several months of symptoms prior to his negative testing ${ }^{[4]}$. Clinically this means one should not quit thinking about PML just because CSF testing is negative. Frequently, it is useful to repeat a lumbar puncture if the clinical suspicion for PML is high, especially given that early infections can test negative. Also, knowing the sensitivity of your lab's JC virus PCR testing is helpful. For example, at the Quest Diagnostics Infectious Disease Lab in California where Duke Neurology typically sends their CSF for JC Virus PCR testing, the test requires more then 500 copies per milliliter in order to be positive. In the end, if the clinical history and imaging are suggestive of PML, a biopsy is the best approach for a definitive diagnosis.

Second, the fact that this patient was found to have sarcoidosis was significant and made the diagnosis very challenging. It was presumed that his symptoms were related to neuro-sarcoidosis because of his history of dermatologic sarcoidosis and his hilar lymph node biopsy that was consistent with sarcoidosis. This was despite the fact that his brain MRI lacked contrast enhancement, which is very atypical for neurosarcoidosis. Very importantly, sarcoidosis is a known risk factor for PML and frequently causes a delay in diagnosis $^{[5]}$. This was definitely the case for patient $\mathrm{D}$, who was treated for neuro-sarcoidosis for months before we decided to do a brain biopsy. The treatment of PML is the opposite of treatment for neurosarcoidosis so quickly reaching the diagnosis is paramount in these cases. This patient needed all immune suppressing therapy stopped, but instead received several treatments with intravenous high dose steroids.

Third, this case report documents a rare case where a patient is only minimally immunosuppressed with hydroxychloroquine and still developed PML. There was only one other case report found in the literature of a patient that developed PML in the setting of being immunosuppressed with only hydroxychloroquine ${ }^{[6]}$. The patient in that case report was a 55-year-old female with lupus. She presented with very similar symptoms of worsening right-sided weakness and aphasia, and her brain MRI looked remarkably similar to that of patient D. Initially there was concern that she may have lupus cerebritis, but all lab testing was negative. Her CSF was positive for JC virus DNA, and brain biopsy confirmed the diagnosis of PML.

The similarity of these two cases makes us suspicious that the hydroxychloroquine was a contributing factor to both patients developing PML. It is possible that the sarcoidosis made our patient susceptible to PML, but typically sarcoidosis causes PML in the setting of immunosuppression with prolonged use of corticosteroids or lymphopenia, and neither was present in this case ${ }^{[7]}$. Our patient was treated with prolonged use of steroids, but that was after his neurological deterioration began, so we did not think this was the cause of his PML (although it may have caused him to worsen). Also, the patient from the other case report was being treated for lupus, did not have sarcoidosis, and lab testing showed that her lupus was not very active during the time she developed PML. It is possible, however, that the hydroxychloroquine was not a contributing factor, and these patients developed PML secondary to sarcoidosis and Lupus respectively as these conditions are known to elevate your risk of $\mathrm{PML}^{[8]}$. It is also known that PML occurs spontaneously in about 1/200,000 patients, so its possible patient D's PML was not related to any of these factors at all ${ }^{[9]}$.

Regardlessly, hydroxychloroquine is frequently prescribed as an immunosuppressive in the setting of rheumatologic illness, and physicians should be aware of this potential complication if they have patient on hydroxychloroquine who is neurologically deteriorating. It is important to identify drugs that could potentially cause PML because the disease is neurologically debilitating and very often fatal. Treatment usually includes withdrawing any offending drugs (using plasmapheresis if necessary) and treating HIV if that is the cause. The goal is to restore immunity to the patient. As the medical community has learned to identify the illness and treat aggressively by restoring immunity or remove the offending agent, outcomes have started to improve with HIV patients having a 2-year mortality of about 50\% (decreased from about $90 \%$ ), and PML patients secondary to natalizumab doing even better ${ }^{[0]}$. There are many other antiviral agents, 
immune response modulators, and immunization strategies currently being developed to treat $\mathrm{PML}^{[10]}$. Since removing the offending agent is an important aspect of effective treatment, it becomes vital to document cases of PML secondary to a drug like hydroxychloroquine, which is not commonly associated with PML. At the very least, future physicians should continue to document cases of PML where hydroxychloroquine may be the cause to try and see if this is more common than previously thought.

\section{DECLARATIONS}

\section{Acknowledgments}

We would also like to acknowledge Dr. Sinha, our program director, for his support.

\section{Authors' contributions}

Helped conceptualize the idea behind this article and cared for the patient in the hospital: Skeen M, Morgenlander J

Compiled all the information and wrote the article: Inamullah O, Farin C, Tran L

Provided the images of pathology: McLendon $\mathrm{R}$

\section{Financial support and sponsorship}

None.

\section{Conflicts of interest}

There are no conflicts of interest.

\section{Patient consent}

Not applicable.

\section{Ethics approval}

Not applicable.

\section{Copyright}

(c) The Author(s) 2018.

\section{REFERENCES}

1. Weber T, Trebst C, Frye S, Cinque P, Vago L, Sindic CJ, Schulz-Schaeffer WJ, Kretzschmar HA, Enzensberger W, Hunsmann G, Lüke W. Analysis of the systemic and intrathecal humoral immune response in progressive multifocal leukoencephalopathy. $J$ Infect Dis 1997;176:250-4.

2. Berger JR, Aksamit AJ, Clifford DB, Davis L, Koralnik IJ, Sejvar JJ, Bartt R, Major EO, Nath A. PML diagnostic criteria: consensus statement from the AAN Neuroinfectious Disease Section. Neurology 2013;80:1430-8.

3. Ikeda J, Matsushima A, Ishii W, Goto T, Takahashi K, Nakamichi K, Saijo M, Sekijima Y, Ikeda SI. Brain biopsy is more reliable than the DNA test for JC virus in cerebrospinal fluid for the diagnosis of progressive multifocal leukoencephalopathy. Intern Med 2017;56:1231-4.

4. Hammarin A, Bogdanovic G, Svedhem V, Pirskanen R, Morfeldt L, Grandien M. Analysis of PCR as a tool for detection of JC virus DNA in cerebrospinal fluid for diagnosis of progressive multifocal leukoencephalopathy. J Clin Microbiol 1996;34:2929-32.

5. Jamilloux Y, Néel A, Lecouffe-Desprets M, Fèvre A, Kerever S, Guillon B, Bouvry D, Varron L, Redares C, Dominique S, Roux M, Chapelon-Abric C, Valeyre D, Ducray F, Bernard C, Broussolle C, Hamidou M, Sève P. Progressive multifocal leukoencephalopathy in patients with sarcoidosis. Neurology 2014;82:1307-13.

6. Sundhar J, Patnaik A, Roppelt H, Mirza N, Marinescu L. Progressive multifocal leukoencephalitis in a patient with systemic lupus erythematosus on hydroxychloroquine. Intern J Rheumatol 2012;7:1-4.

7. Pallin M, O’Sullivan C, Dodd J, McCreery K, Brett F, Farrell M, O’Brien D, Hall WW, Tubridy NJ, Keane MP. A case of progressive multifocal leukoencephalopathy in a patient with sarcoidosis. QJM 2012;105:1011-6.

8. Calabrese LH, Molloy ES. Progressive multifocal leucoencephalopathy in the rheumatic diseases: assessing the risks of biological immunosuppressive therapies. Ann Rheum Dis 2008;67 Suppl 3:iii64-5. 
9. Progressive Multifocal Leukoencephalopathy. National Organization for Rare Disorders. 2015. Available from: https://rarediseases.org/ rare-diseases/progressive-multifocal-leukoencephalopathy [Last accessed on 30 Jan 2018]

10. Pavlovic D, Patera AC, Nyberg F, Gerber M, Liu M; Progressive Multifocal Leukeoncephalopathy Consortium. Progressive multifocal leukoencephalopathy: current treatment options and future perspectives. Ther Adv Neurol Disord 2015;8:255-73. 